\title{
Comportamiento de las listas de distribución de documentación de RedIRIS durante la década 2002-2011
}

An analysis of RedIRIS Information Science mailing lists from 2002 to 2011

\author{
Ana María CoRdero-GonZÁlez, Cristina FABA-PÉREZ \\ Departamento de Información y Comunicación, Facultad de Ciencias de la Documentación y la Comunicación, Universidad de \\ Extremadura \{amcorgon|cfabper@alcazaba.unex.es\}
}

\begin{abstract}
Resumen
Se analiza la conducta de las listas de distribución que forman parte de la categoría Documentación de RedIRIS de 2002 a 2011 teniendo en cuenta dos variables: la evolución temporal del número de mensajes enviados; y el comportamiento de los dominios de las direcciones de correo electrónico de origen. Los resultados demuestran que los mensajes de correo electrónico enviados a las listas experimentaron un crecimiento del $28,45 \%$ en el periodo analizado y que los dominios de correo de instituciones científico-educativas se mantuvieron a lo largo de toda la década con porcentajes superiores a los dominios personales o comerciales, llegando a incrementarse en un $4,27 \%$ durante la década. Estos resultados confirman la importancia del correo electrónico como un medio de comunicación muy útil y fiable entre la comunidad científica y educativa relacionada con la Documentación en España.
\end{abstract}

Palabras clave: Listas de distribución. Documentación. Evolución temporal. Dominios de correo electrónico. España.

\section{Introdución}

Según palabras de Merlo Vega y Sorli Rojo (1999),

Una lista de distribución es una agrupación de usuarios de correo electrónico que basa su trabajo en un software de envío simultáneo de mensajes, de modo que todos los suscriptores de un grupo reciben cada mensaje remitido por cualquiera de los participantes al servidor en el que se encuentra instalado este programa de difusión masiva de correos electrónicos.

Es decir, una lista de distribución (también conocida como lista de correo, lista de discusión, foro de discusión o grupo de discusión) es un conjunto de direcciones de correo electrónico que se usan para enviar ciertos mensajes o anuncios con un contenido de interés general para todos los miembros de la lista. Dichos miembros pueden ocupar el role de administrador-moderador, quien se encarga del buen fun-

\begin{abstract}
The behavior of the Information Science mailing lists hosted in the Spanish universities' RedIRIS service during the decade 2002-2011 is analysed according to two variables: the evolution of the number of messages sent to the distribution lists, and the email addresses domains of the authors. The results show that e-mail messages sent to the lists increased by $28.45 \%$ and that the mail domains of scientific and educational institutions have higher percentages than the personal or commercial mail domains, increasing by $4.27 \%$ during the period. These results confirm the importance of email as a very useful and reliable communication medium for the Spanish scientific and educational LIS community.
\end{abstract}

Keywords: Distribution lists. Documentation. Temporal evolution. E-mail domains. Spain.

cionamiento de la lista, o de usuario, quien puede recibir y enviar correos a la lista. Algunas listas son abiertas y puede suscribirse quien lo desee, y otras cerradas, es decir, limitadas a aquellas personas que cumplan una serie de requisitos, como pertenencia a un grupo profesional, interés demostrado por la temática de la lista, etc.

El incremento de las listas de distribución desde su aparición ha sido vertiginoso. La primera lista nacía en 1975, siete años después había 44 listas registradas y hoy en día existen millones en todo el mundo (García Aretio, 2012). Desde su comienzo surgieron multitud de listas de uso interno entre departamentos, grupos de investigación con proyectos en marcha o, simplemente, personas con intereses comunes (Fernández Merino, Maceiras García y Zurriaga Lloréns, 2000). Desde su nacimiento, tanto las listas de distribución como los correos electrónicos que las conforman, han sido estudiados desde dife- 
rentes puntos de vista, entre los que se pueden mencionar a modo de ejemplo: la interacción que permiten las listas de distribución en procesos de educación a distancia de tipo asincrónico (Rinaudo, Chiecher y Donolo (2002); su uso como técnica de marketing en línea al permitir conocer los intereses de los clientes (Corredera, 2003); la relación de las listas de distribución con las redes sociales (Márquez Espino, 2010; García Aretio, 2012); el uso del correo electrónico para compartir información científica (Hamner, Yip y Dessy, 1994; Herrero Solana, 1996); el comportamiento del correo electrónico en diferentes ámbitos como el universitario (Satoh, 1997; Capra, Khanova y Ramdeen, 2013); la seguridad y privacidad de los sistemas de correo electrónico certificados (Tauber y Rössler, 2011); o los efectos secundarios indeseables pero inevitables (correos basura y virus) que conlleva su uso (Fernández Merino, Maceiras García y Zurriaga Lloréns, 2000).

En la presente investigación, se ha profundizado en las listas de distribución que forman parte de la categoría Documentación y se han estudiado desde dos puntos de vista o variables diferentes: a) la evolución temporal de los mensajes enviados a las listas de distribución, que pueden llevarlas al fracaso o a su consolidación definitiva; y b) el comportamiento de los dominios de las direcciones de correo electrónico desde las que se envían los mensajes a las listas.

Con respecto a la primera variable, Nagel (1994) estableció el ciclo de vida natural de las listas con una primera etapa de entusiasmo inicial, a la que seguiría otra de evangelización (proselitismo). La tercera etapa estaría caracterizada por la expansión de la lista; continuada por otra en la que se establecen vínculos de colaboración entre los suscriptores. A partir de aquí se produciría la fase de disconformidad con la diversidad y comienzan los problemas por el aumento de mensajes o el desencanto de los suscriptores. La fase final puede derivar bien en la autocomplacencia y el consiguiente estancamiento o bien en la madurez de la lista -0 , lo que es lo mismo, su afianzamiento definitivo-. En realidad, el ciclo de vida de las listas de distribución se puede considerar idéntico al de cualquier otro producto y, según Kotler (2002) se compone de cuatro etapas: introducción, crecimiento, madurez y decrecimiento.

Con respecto a la segunda variable, el dominio de las direcciones de correo electrónico, ha sido analizada por algunos autores como Garfinkel (2002). Como ya es conocido, la parte derecha del símbolo arroba @ de una dirección de correo electrónico corresponde al dominio e identifica, a menudo, a un servidor en particular, que pertenece a alguna organización. Para identificar a cuál, se divide la cadena situada a la derecha del símbolo @ en subdominios separados entre sí por puntos. Aquél subdominio que está situado más a la derecha se conoce como top level domain (TLD) o dominio de más alto nivel y puede ser geográfico (por ejemplo, es de España, .fr de Francia, etc.) o funcional (por ejemplo, .edu para centros educativos, .com para información comercial y empresas particulares, .org para organizaciones sin ánimo de lucro, asociaciones o fundaciones, .net originalmente para empresas relacionadas con Internet y la tecnología, etc.). El siguiente ejemplo sirve para ilustrar la sintaxis general de una dirección de correo electrónico:

\begin{tabular}{|c|c|}
\hline Nombre del Buzón: & $a b c$ \\
\hline Subdominio2: & alcazaba \\
\hline Subdominio1: & unex \\
\hline TLD: & es \\
\hline \multicolumn{2}{|c|}{$\begin{array}{l}\text { Esta dirección describe el buzón de correo de un usuario } \\
\text { cuyo identificador es 'abc'. Dicho buzón se encuentra ubica- } \\
\text { do en un servidor llamado 'alcazaba', que pertenece a la } \\
\text { Universidad de Extremadura 'unex' que se encuentra dentro } \\
\text { del dominio de más alto nivel de España 'es'. }\end{array}$} \\
\hline
\end{tabular}

Figura 1. Ejemplo de una dirección de correo electrónico

Así pues, el objetivo del presente trabajo consiste en analizar las listas de distribución que pertenecen a la categoría Documentación a través de dos variables: la evolución temporal de los mensajes enviados a las listas, y el comportamiento de los dominios de las direcciones de correo electrónico de los mensajes enviados a las mismas.

\section{Fuentes y metodología}

Los servidores que albergan las listas de distribución suelen pertenecer a instituciones científicas o académicas, que destinan un ordenador en exclusiva para tal fin. En España destaca el servidor LISTSERV ${ }^{1}$ de RedIRIS que es la red académica y de investigación española que proporciona servicios avanzados de comunicaciones a la comunidad científica y universitaria nacional. Está financiada por el Ministerio de Economía y Competitividad, y gestionada por la entidad pública empresarial Red.es, del Ministerio de Industria, Energía y Turismo ${ }^{2}$.

\footnotetext{
http://www.rediris.es/list/

2 http://www.rediris.es/rediris/
} 
Las listas de distribución de LISTSERV se agrupan en 30 categorías temáticas. La categoría escogida para este trabajo es Documentación, formada por 32 listas. Como la etapa escogida para realizar el análisis es la década que se extiende desde 2002 hasta 2011, solo se han tenido en cuenta aquellas listas operativas o "vivas" durante dicho periodo, sin juzgar si la lista está bien ubicada en la categoría o no. En total se trabaja con 15 listas (tabla I). El motivo por el que se han seleccionado las listas de distribución de LISTSERV es la oficialidad de dicha fuente.

\begin{tabular}{|c|c|c|}
\hline $\begin{array}{l}\text { Lista de } \\
\text { distribución }\end{array}$ & Descripción & $\begin{array}{l}\text { Año de } \\
\text { creación }\end{array}$ \\
\hline aha & $\begin{array}{l}\text { Lista de la Asociación de Historia } \\
\text { Actual }\end{array}$ & 2002 \\
\hline arxiforum & Foro sobre Archivística & 1998 \\
\hline bescolar & $\begin{array}{l}\text { Coordinación de Bibliotecas } \\
\text { Escolares }\end{array}$ & 1999 \\
\hline bib-med & $\begin{array}{l}\text { Documentación en Medicina y } \\
\text { Ciencias de la Salud }\end{array}$ & 1995 \\
\hline cinedoc & $\begin{array}{l}\text { Documentación y Nuevas } \\
\text { Tecnologías en el Cine Español }\end{array}$ & 1998 \\
\hline fidel & $\begin{array}{l}\text { Fuentes de Información y } \\
\text { Documentación en Línea }\end{array}$ & 1998 \\
\hline harte-I & Foro sobre Historia del Arte & 2000 \\
\hline isko-es & $\begin{array}{l}\text { Conocimiento en Sistemas de } \\
\text { Información y Documentación }\end{array}$ & 1997 \\
\hline issi & $\begin{array}{l}\text { Scientometrics, Informetrics and } \\
\text { Cybermetrics }\end{array}$ & 2001 \\
\hline iwetel & $\begin{array}{l}\text { Foro para Profesionales de } \\
\text { Bibliotecas y Documentación }\end{array}$ & 1993 \\
\hline medired & Fuentes de Información Médicas & 2001 \\
\hline musicdoc & $\begin{array}{l}\text { Documentación sobre Música y } \\
\text { Musicología }\end{array}$ & 1998 \\
\hline recida & $\begin{array}{l}\text { Red de Centros de Información y Red } \\
\text { de Centros de Información y } \\
\text { Documentación Ambiental }\end{array}$ & 2002 \\
\hline reder & $\begin{array}{l}\text { Red de Estudios y Difusión del Exilio } \\
\text { Republicano de } 1939\end{array}$ & 2000 \\
\hline virtuarte & Foro del Mundo del Arte & 1999 \\
\hline
\end{tabular}

Tabla I. Listas de distribución vivas durante 20022011 de la categoría Documentación

Para recopilar los mensajes de correo electrónico enviados a las listas de distribución y comprobar su evolución temporal y el comportamiento de sus dominios, previamente ha sido preciso registrarse y suscribirse a cada una de las 15 listas seleccionadas y, una vez aceptada la suscripción, se han analizado los archivos de cada lista. En dichos archivos, los mensajes enviados a las listas se clasifican por meses, e incluso por semanas, por lo que para cada una de las 15 listas se ha generado una matriz de 10 años por 12 meses, donde el contenido de cada celdilla se corresponde con el total de mensajes recopilados manualmente (véase, a modo de ejemplo, la tabla II con los mensajes enviados a la lista ISSI). EI número total de mensajes recopilados en las 15 listas de la categoría Documentación ha sido 59.959 (véase tabla IV).

\begin{tabular}{|c|c|c|c|c|c|c|c|c|c|c|}
\hline & §ิ & 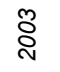 & ষ্ণ & $\stackrel{\overbrace{}}{\stackrel{ి}{ి}}$ & ঠి & \&ి & $\stackrel{\infty}{\stackrel{\sim}{~}}$ & 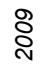 & $\stackrel{\circ}{\text { D }}$ & $\stackrel{i}{\text { D }}$ \\
\hline Ener. & 0 & 13 & 11 & 4 & 3 & 8 & 5 & 5 & 5 & 11 \\
\hline Febr. & 1 & 14 & 3 & 6 & 3 & 5 & 5 & 8 & 4 & 10 \\
\hline Marz. & 8 & 14 & 11 & 8 & 6 & 11 & 6 & 11 & 9 & 9 \\
\hline Abril & 1 & 11 & 11 & 6 & 6 & 8 & 4 & 9 & 3 & 1 \\
\hline May. & 1 & 8 & 8 & 2 & 5 & 9 & 6 & 12 & 4 & 5 \\
\hline Jun. & 8 & 11 & 8 & 7 & 5 & 7 & 7 & 6 & 17 & 8 \\
\hline Julio & 5 & 10 & 11 & 6 & 3 & 3 & 6 & 4 & 13 & 3 \\
\hline Agos. & 8 & 9 & 5 & 7 & 3 & 6 & 2 & 8 & 3 & 3 \\
\hline Sept. & 6 & 13 & 7 & 12 & 9 & 4 & 6 & 3 & 2 & 8 \\
\hline Oct. & 5 & 10 & 8 & 0 & 6 & 7 & 9 & 4 & 4 & 7 \\
\hline Nov. & 5 & 6 & 8 & 4 & 7 & 8 & 12 & 6 & 5 & 4 \\
\hline Dic. & 5 & 5 & 2 & 4 & 13 & 7 & 2 & 7 & 3 & 5 \\
\hline Total & 53 & 124 & 93 & 66 & 69 & 83 & 70 & 83 & 72 & 74 \\
\hline
\end{tabular}

Tabla II. Mensajes enviados a la Lista de distribución ISSI en la década 2002-2011

\begin{tabular}{|c|c|c|}
\hline $\begin{array}{l}\text { Tipo de dominio- } \\
\text { mensajes ISSI }\end{array}$ & Año 2002 & $\begin{array}{c}N^{\circ} \text { de } \\
\text { mensajes }\end{array}$ \\
\hline \multirow{11}{*}{$\begin{array}{l}\text { Científico } \\
\text { Educativo }\end{array}$} & @POSTA.UNIZAR.ES & 7 \\
\hline & @UNSW.EDU.AU & 7 \\
\hline & @USAL.ES & 6 \\
\hline & @CINDOC.CSIC.ES & 2 \\
\hline & $@$ @DE.ES & 2 \\
\hline & @CSCD.AC.CN & 2 \\
\hline & @SOLENT.AC.UK & 2 \\
\hline & @ANU.EDU.AU & 1 \\
\hline & @EMAIL.ARIZONA.EDU & 1 \\
\hline & @METU.EDU.TR & 1 \\
\hline & @NIWI.KNAW.NL & 1 \\
\hline \multirow{7}{*}{$\begin{array}{l}\text { Personal } \\
\text { Comercial }\end{array}$} & @LEYDESDORFF.NET & 12 \\
\hline & @TERRA.COM.PE & 3 \\
\hline & @MSN.COM & 2 \\
\hline & @YAHOO.COM & 1 \\
\hline & @STAR-GROUP.NET & 1 \\
\hline & @EARTHLINK.NET & 1 \\
\hline & @T-ONLINE.DE & 1 \\
\hline Total & & 53 \\
\hline
\end{tabular}

Tabla III. Tipos de dominios de los mensajes enviados a ISSI en 2002 
Además, en cada mensaje, se ha anotado el dominio completo de la dirección de correo electrónico con el fin de analizar su comportamiento. Para ello, se han diseñado dos grandes categorías: dominios de instituciones científicas o educativas y dominios personales o de instituciones comerciales; y se ha ido clasificando cada dirección en su categoría correspondiente una vez comprobada su procedencia. Como ejemplo, en la tabla III, en la página anterior, se muestran los dominios de los mensajes enviados a la lista ISSI en 2002.

\section{Resultados}

Para ver la evolución temporal de los mensajes enviados a las listas de distribución de la categoría Documentación durante la década analizada, la tabla IV y el gráfico 1 muestra el número total de mensajes recopilados en las 15 listas de distribución de dicha categoría (59.959 mensajes) ordenados descendentemente por el total de mensajes por lista.

\begin{tabular}{lcccccccccccc}
\hline Listas documentación & 2002 & 2003 & 2004 & 2005 & 2006 & 2007 & 2008 & 2009 & 2010 & 2011 & Total & $\%$ \\
\hline iwetel & 1825 & 2243 & 2172 & 2765 & 1926 & 2090 & 2066 & 2222 & 3204 & 3214 & 23727 & 39,57 \\
\hline arxiforum & 1005 & 1239 & 1391 & 1269 & 1422 & 1212 & 1319 & 1382 & 1364 & 1546 & 13149 & 21,93 \\
\hline bib-med & 829 & 679 & 619 & 466 & 401 & 379 & 393 & 436 & 435 & 435 & 5072 & 8,46 \\
\hline fidel & 445 & 384 & 390 & 344 & 432 & 413 & 421 & 304 & 331 & 424 & 3888 & 6,48 \\
\hline recida & 56 & 72 & 67 & 54 & 187 & 370 & 393 & 421 & 474 & 449 & 2543 & 4,24 \\
\hline bescolar & 114 & 55 & 127 & 250 & 352 & 394 & 317 & 180 & 199 & 258 & 2246 & 3,75 \\
\hline aha & 95 & 144 & 299 & 314 & 227 & 269 & 242 & 190 & 171 & 193 & 2144 & 3,58 \\
\hline reder & 369 & 227 & 197 & 179 & 158 & 142 & 132 & 65 & 64 & 56 & 1589 & 2,65 \\
\hline harte-I & 190 & 113 & 119 & 73 & 125 & 101 & 336 & 144 & 123 & 122 & 1446 & 2,41 \\
\hline isko-es & 152 & 158 & 155 & 120 & 136 & 148 & 117 & 100 & 124 & 169 & 1379 & 2,30 \\
\hline medired & 167 & 155 & 144 & 72 & 41 & 24 & 39 & 47 & 25 & 84 & 798 & 1,33 \\
\hline issi & 53 & 124 & 93 & 66 & 69 & 83 & 70 & 83 & 72 & 74 & 787 & 1,31 \\
\hline virtuarte & 205 & 83 & 41 & 35 & 48 & 26 & 11 & 3 & 19 & 43 & 514 & 0,86 \\
\hline musicdoc & 12 & 108 & 70 & 74 & 65 & 54 & 31 & 12 & 22 & 26 & 474 & 0,79 \\
\hline cinedoc & 8 & 29 & 27 & 4 & 6 & 84 & 36 & 5 & 0 & 4 & 203 & 0,34 \\
\hline Total & 5525 & 5813 & 5911 & 6085 & 5595 & 5789 & 5923 & 5594 & 6627 & 7097 & 59959 & 100 \\
\hline
\end{tabular}

Tabla IV. Mensajes enviados a las Listas de distribución de la categoría Documentación en la década 2002-2011

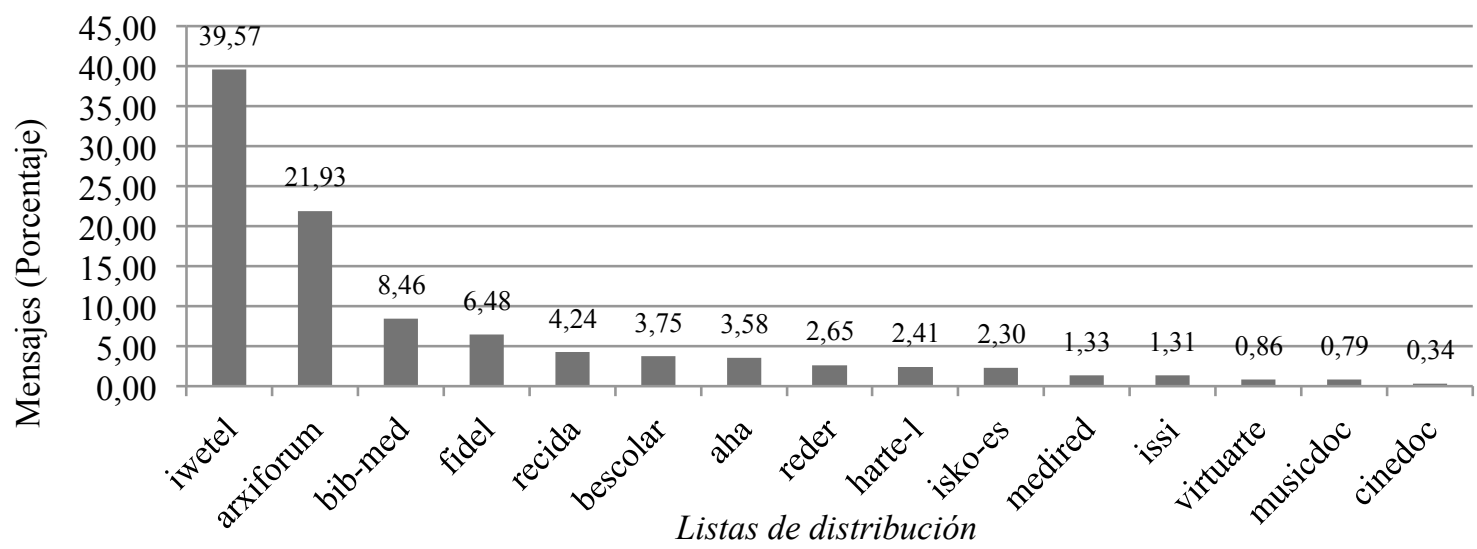

Gráfico 1. Actividad de las Listas de distribución de la categoría DOCUMENTACIÓN en la década 2002-2011

En el gráfico 1 se observa que la lista de distribución Iwetel, en primer lugar, y Arxiforu, en segunda posición, destacan sobre el resto, acumulando el $61,50 \%$ del total. Como la fecha de creación de ambas listas es temprana (1993 y 1998, respectivamente) se podría pensar que este comportamiento es lógico puesto que a mayor antigüedad de la lista, mayor probabilidad 
de que sea utilizada y, por consiguiente, mayor número de mensajes. Sin embargo, hay que matizar que cada lista de distribución tiene su propia idiosincrasia. Así, en el gráfico 1 se comprueba que Iwetel sí cumple esta premisa, puesto que es la lista más antigua (1993) y más activa (primera posición); pero, por ejemplo, Iskoes, nacida en 1997, ocupa el puesto décimo. Y las listas creadas en 1998 ocupan posiciones extremas: Arxiforum y Fidel se sitúan en la se- gunda y cuarta posición, respectivamente; mientras que Cinedoc y Musicdoc se ubican en la última (decimoquinta) y penúltima posición (decimocuarta), respectivamente. Por otro lado, listas nacidas con posterioridad y que, hipotéticamente, habrían tenido menos tiempo para afianzarse, ocupan buenas posiciones en la distribución. Este es el caso de Recida y Aha nacidas en 2002 y que se sitúan respectivamente en las posiciones quinta y séptima.

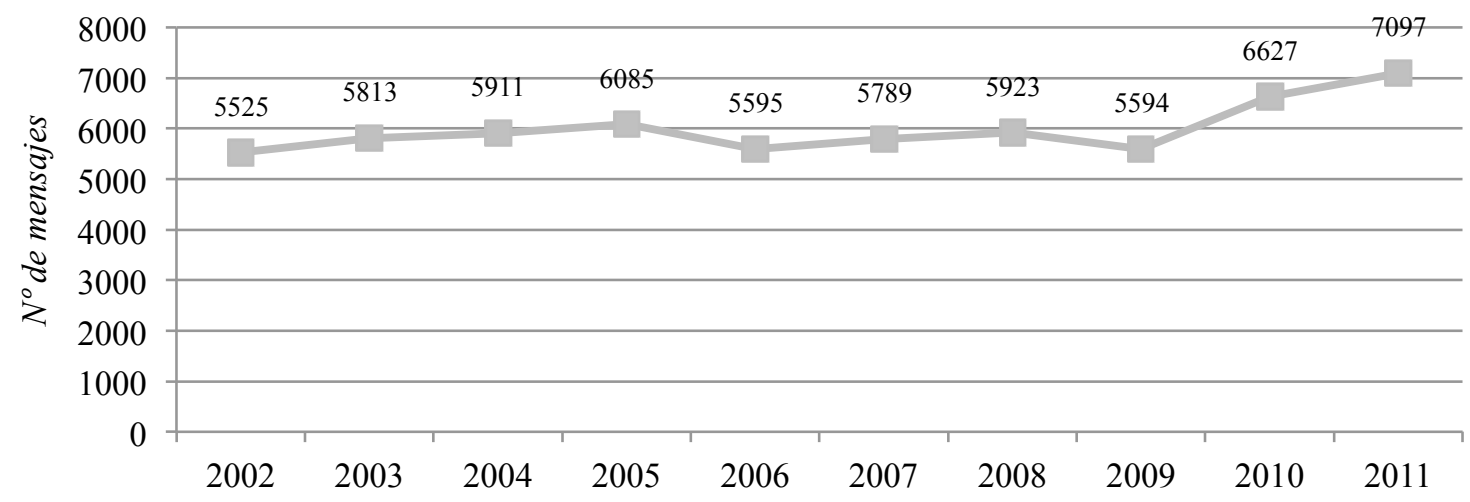

Gráfico 2. Evolución de los mensajes enviados

En el gráfico 2 se observa la evolución temporal de las listas agrupadas en la categoría Documentación durante 2002-2011. Aunque la tendencia general es positiva con un incremento en 2011 respecto a 2002 del $28,45 \%$, se observa un decrecimiento significativo en 2006 con res- pecto a 2005, sin duda, porque Iwetel decrece en 839 mensajes, y en 2009 con respeto a 2008 debido al decrecimiento de Harte-I con 192 mensajes menos, Bescolar que desciende en 137 mensajes, y Fidel que baja en 137.

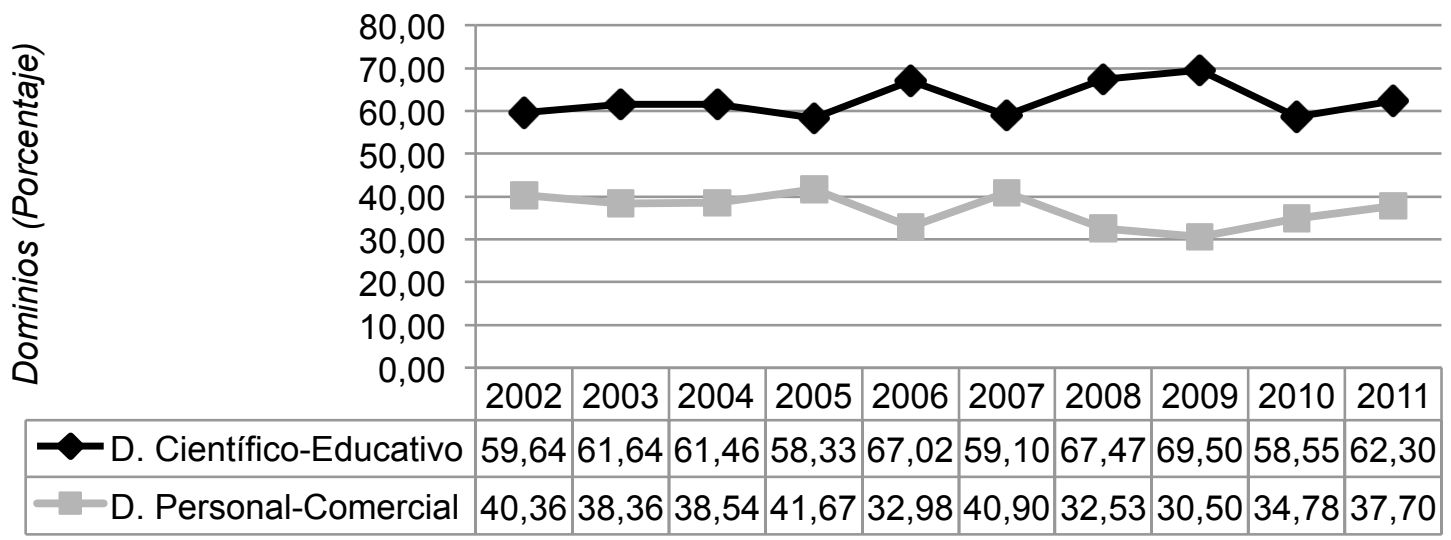

Gráfico 3. Comportamiento de los dominios de las direcciones de correo electrónico de las Listas de distribución de la categoría DOCUMENTACIÓN en la década 2002-2011

Con respecto al comportamiento de los dominios de las direcciones de correo electrónico de los mensajes enviados a las listas de distribución de Documentación, el gráfico 3 representa el promedio de dominios de cada categoría elegida (dominios de instituciones científicas o educativas, y dominios personales o de institu- ciones comerciales) en cada año. Así, por ejemplo, el año 2002 , el $59,64 \%$ de los dominios correspondían a instituciones científico-educativas frente al 40,36\% de dominios personales e instituciones comerciales. En general, a lo largo de toda la década, se observa una mayor proporción de dominios del tipo científico-educativo, 
con un incremento en 2011 con respecto a 2002 del $4,27 \%$. Los dominios personales-comerciales, sin embargo, sufren un decrecimiento del $6,58 \%$ en 2011 con respecto a 2002. También se observa un comportamiento muy homogéneo durante toda la década que oscila entre el $58,33 \%$ en 2005 y $69,5 \%$ en 2009 en el caso de los dominios científico-educativos; $y$, por consiguiente, entre el $41,67 \%$ en 2005 y el $30,5 \%$ en 2009 de los personales-comerciales.

\section{Conclusiones}

Se ha discutido mucho acerca de la influencia que los nuevos sistemas de comunicación (redes sociales, mensajería instantánea, blogs, etc.) han tenido en el descenso del uso del correo electrónico y, por analogía, en la utilización de las listas de distribución. Según datos de Nielsen Company (s. a.; citado en Márquez Espino, 2010) el tiempo dedicado al correo electrónico descendió en un $28 \%$ en 2010 con respecto al año anterior. Sin embargo, con el presente trabajo se ha demostrado que los mensajes de correo electrónico enviados a las listas de distribución de la categoría Documentación han experimentado un crecimiento a lo largo de la década 2002-2011 del 28,45\%. Este incremento corrobora la teoría de que el uso del correo electrónico sigue siendo una forma de comunicación muy extendida en la Red. De hecho, el uso del correo electrónico se puede considerar más útil que otros medios de comunicación, como las redes sociales, cuando el mensaje que se quiere transmitir contiene información relevante o es necesario que quede constancia de la misma.

Además, el resultado obtenido con los dominios de correo electrónico de instituciones científicoeducativas que se han mantenido a lo largo de toda la década con porcentajes superiores a los dominios personales o comerciales, demuestra la importancia del correo electrónico como un medio de comunicación extremadamente útil y fiable entre la comunidad científica y educativa del mundo relacionado con la Documentación. Esta tipología de dominio ha llegado incluso a incrementarse en un $4,27 \%$ en 2011 con respecto a 2002.

Por otro lado, se ha comprobado que la antigüedad de cada lista y su número de mensajes no es directamente proporcional (a mayor antigüedad, mayor número de mensajes), exceptuando el caso sobresaliente de Iwetel (Foro para Profesionales de Bibliotecas y Documentación) que ha demostrado ser la más longeva (1993) y a la vez la más activa de la categoría analizada.

\section{Agradecimientos}

Este trabajo ha sido financiado por la Junta de Extremadura, Consejería de Empleo, Empresa e Innovación y el Fondo Social Europeo como parte de la ayuda a grupos GR10019.

\section{Referencias}

Capra, R., Khanova, J.; Ramdeen, S. (2013). Work and personal e-mail use by university employees: PIM practices across domain boundaries. // Journal of the American Society for Information Science and Technology. 64:5 (2013) 1029-1044.

Corredera, L. (2003). Creación y gestión de listas de distribución. // Hipertext.net, 1 (2003) 14.

Fernández Merino, J.C., Maceiras García, L.; Zurriaga Llorens, O. (2000). La discusión electrónica o qué son las listas de distribución. // Gaceta Sanitaria. 14:4 (2000) 314-317.

Herrero Solana, V.F. (1996). La utilización de foros de discusión electrónicos como fuentes de información sobre la comunidad científica informal. // Revista General de Información y Documentación. 6:2 (1996) 219-229.

García Aretio, Lorenzo (2012). ¿Listas de distribución vs Software social?. // Revista Iberoamericana de Educación a Distancia (RIED). 15.2 (2012). http:. // http://aretio.hypotheses.org/92 (02-02-2013).

Garfinkel, S. (2002). Declare e-mail independence. // Technology Review. 105:6 (2002) 29.

Hamner, V., Yip, C.-W.; Dessy, R.E. (1994). E-mail as a tool for sharing binary files among scientists. // Journal of Chemical Information and Computer Sciences. 34:3 (1994) 480-484.

Kotler, Philip. (2002). Dirección de marketing. Conceptos esenciales. México: Pearson Educación, 2002.

Márquez Espino, Emilio (2010). Las redes sociales y el correo electrónico: ¿enemigos?. // Puro marketing.com (19-08-2010). http://www.puromarketing.com/42/7779/re des-sociales-correo-electronico-enemigos.html (02-032013).

Merlo Vega, José Antonio; Sorli Rojo, Ángela (1999). Las listas de distribución como herramienta profesional. II Métodos de Información. (Julio 1999). http://exlibris .usal.es/merlo/escritos/pdf/mei.pdf (20-03-2013).

Nielsen Company. http://www.nielsen.com/.

Nagel, K. (1994) The natural life cycle of mailing lists. http://users.rider.edu/ suler/psycyber/lifelist.html (23-052013).

Rinaudo, M.C.; Chiecher, A.; Donolo, D. (2002). Las listas de distribución como espacios de interacción entre tutores; alumnos. // Revista de Educación a Distancia. 2 (2002).

Satoh, N. (1997). Analysis of the recent use of electronic mail at the University of Occupational and Environmental Health, Japan. // Journal of UOEH. 19:1 (1997) 39-48.

Tauber, A.; Rössler, T. (2011). Enhancing security and privacy in certified mail systems using trust domain separation. // Lecture Notes in Computer Science (including subseries Lecture Notes in Artificial Intelligence and Lecture Notes in Bioinformatics), 6531 LNCS (2011) 152158.

Enviado: 2013-06-09. Segunda versión: 2013-07-04. Aceptado: 2013-08-27. 